\title{
LAS CLASES MEDIAS: CRITERIOS DE DEFINICION
}

\author{
Carlota Solé \\ Departamento de Sociología de la Facultad de CC.PP. y Sociología. \\ Universidad Autónoma de Barcelona
}

RESUMEN. Al abordar la realidad de la estructura social de las sociedades contemporáneas, en especial las denominadas sociedades avanzadas, deben matizarse los planteamientos de "suma cero" o dicotómicos. Es más adecuado analizar esa realidad bajo un prisma estratificacional o gradacional más que relacional. Así, se ajusta más a la compleja estructura social de estas sociedades hablar de estratos en una estructura de ocupaciones, de poder y de autoridad, de privilegio o de prestigio, que de clases sociales antagónicas en términos excluyentes. Las mismas relaciones entre los grupos sociales (sean clases, grupos de intereses, élites, etc.) combinan distintos criterios de definición. Existen múltiples situaciones intermedias de control sobre lo medios de producción y asignación de recursos, y múltiples posiciones sociales sobrepuestas.

\section{Explotación, dominación o restricción excluyente}

El concepto de clase social fue establecido, teórica e históricamente, por Karl Marx y fue aceptado universalmente como punto de partida de posteriores elaboraciones teóricas sobre la desigualdad social, así como de investigaciones empíricas sobre la estructura social de una sociedad en concreto. Sin embargo, el concepto marxista de clase, con ser el más universalmente aceptado y haber sido tomado como punto de referencia por otros autores clásicos como Weber para elaborar su esquema tridimensional de la estratificación social, conlleva la formulación de un modelo dicotómico de estructura social por el cual se definen con mayor precisión las clases sociales extremas (capitalistas-burgueses-trabajadores-proletarios) 
que las clases intermedias o capas medias. En realidad, Marx dio los elementos teóricos para definir una y otra clases antagónicas limitándose a enunciar en sentido histórico la existencia de clases intermedias o capas medias que acabarían siendo absorbidas por el proletariado ${ }^{1}$. En la época en que escribe Marx ese análisis se corresponde con la realidad y la prospección parece ajustada. Los cambios y transformaciones posteriores en las sociedades industriales que este gran clásico del pensamiento social examina, hacen variar, no obstante, la percepción sobre la precisión y utilidad de los instrumentos conceptuales de análisis. Ya en Weber, pero sobre todo en Dahrendorf, se explicitan los rasgos de la nueva sociedad industrial del siglo XX y la necesidad de conceptualizar teóricamente las llamadas clases medias.

Los rasgos de la nueva sociedad industrial que, en términos de Dahrendorf, seguía al desarrollo del capitalismo son fundamentalmente tres. En primer lugar se produce la división entre la propiedad y el control de la producción (que en las sociedades capitalistas coinciden). Esta característica da lugar a una trasformación o cambio en la estructura de posiciones sociales y la necesidad de reclutar personas que ocupen esas posiciones. De ahí la subsiguiente aparición de las clases medias, compuestas por personas con capacidades y habilidades para controlar el proceso productivo. Poseer o no poseer los medios de producción no es definitorio de la situación de ese grupo de personas ante el proceso de producción, sino su capacidad de control a través del conocimiento y la puesta en práctica de habilidades. El criterio de propiedad no define, de forma determinante, a las clases medias. Es preciso hallar nuevos criterios para definirlas e instrumentalizar el concepto teórico elaborado.

Un segundo rasgo de las sociedades industriales es el de la separación entre conflicto industrial y político en cuanto que el primero no se superpone al segundo, ni deriva un conflicto en la esfera industrial en conflicto entre clases sociales. El papel del Estado como una unidad de decisión económica, más que representante de los intereses de la burquesía, es un factor decisivo en la separación de la esfera industrial y política y en la desaparición del enfrentamiento entre capitalistas y obreros. Consecuencia de ello es el aislamiento de la industria respecto de la sociedad. Este tercer rasgo es consecuencia tanto de la complejidad que adquiere el proceso productivo en las sociedades industriales que siguen al desarrollo del capitalismo (nuevas necesidades, incremento de la demanda de bienes cada vez más diversificados cualitativamente, etc.) como a la necesidad del intervencionismo estatal en todas las esferas de la vida social.

Simplificando, podrían enumerarse las trasformaciones que aparecen en la sociedad industrial respecto a la sociedad capitalista del siglo XIX de la

I K. MARX (1868), Capital; ed. inglesa de Lawrence \& Wishart, Londres, 1970; 18 de Brumario, Ricardo Aguilera, Madrid, 1970. 
siguiente forma: no se produce el proceso de homogeneización de las clases antagónicas entre sí, aparece la "nueva clase media" vinculada a la progresiva asalarización de las clases trabajadoras, el conflicto industrial queda institucionalizado a través de la existencia de potentes organizaciones (sindicatos) que representan los intereses de los trabajadores ${ }^{2}$.

La existencia de los grupos-de-intereses se explica en virtud de las distintas actividades que desarrollan los individuos acordes a las ocupaciones que consignan en el proceso productivo y al control diferencial que mantienen sobre ese proceso. En realidad, Dahrendorf apunta a la distribución desigual de autoridad (en el sentido weberiano del término) en una sociedad (o, en concreto, en toda asociación imperativamente coordinada) como un fenómeno más general y global que el de la propiedad de los medios de producción. De ahí que no sólo en la esfera societal, sino en la organizacional, pueda hablarse de grupos o clases (en un sentido más limitado y parcial que el marxiano) dominante y subordinado, de acuerdo con la distribución diferenciada de autoridad y, por lo tanto, de control sobre el proceso de producción.

El acento puesto en el sistema distributivo más que en el productivo es enfatizado por Parkin al señalar como determinante del mismo el proceso por el cual las colectividades sociales tienden a maximizar sus beneficios, gratificaciones o recompensas restringiendo el acceso a los medios y oportunidades para alcanzarlos a un círculo de elegidos, excluyendo a los outsiders $^{3}$. La restricción de oportunidades económicas y sociales a un núcleo, de dimensiones variables, a fin de monopolizar determinadas oportunidades, generalmente de naturaleza económica, se justifica por cualquier atributo del grupo (la raza, la lengua, el origen social, la religión). Ampliando la elaboración de Weber sobre los procesos de exclusión derivados de la distribución de poder, Parkin toma la idea de restricción social (social closure) para englobar en ella toda suerte de acción social colectiva orientada a maximizar las recompensas y oportunidades. Las estrategias de resțicción incluyen no sólo a las de exclusión, sino también a aquellas adoptadas por quienes están excluidos como respuesta directa a su status como outsiders.

El rasgo distintivo de la restricción excluyente es el de intentar asegurarse por parte de un grupo su posición privilegiada a expensas de otro grupo, a través de un proceso de subordinación. Es una forma de acción colectiva que, intencionadamente o no, da lugar a la categoría social de los no-elegidos o outsiders. Siguiendo a Parkin, la restricción excluyente significa el uso del poder en la dirección «hacia abajon, puesto que implica la

2 R. DAhrendorf (1959), Class and Class Conflict in Industrial Societies, Routledge and Kegan Paul, Londres (1957), pp. 36-72.

${ }_{3}$ F. PARKIN (1979), The Marxist Theory of Class: A Bourgeois Critique, Tavistock, Londres, pp. 44-46. 
creación de un grupo, clase o estrato compuesto por quienes legalmente se definen como inferiores. Por otra parte, la acción contrapuesta por parte de los no-privilegiados significa el uso del poder en la dirección "hacia arriba", en el sentido de que los intentos colectivos de los excluidos para ganar una mayor participación en los recursos siempre supone un recorte de los privilegios de quienes se definen legalmente como superiores. En otras palabras, es una forma de acción orientada hacia el objetivo de la usurpación. La exclusión y la usurpación deben contemplarse como los dos tipos generales más importantes de restricción social, el último siendo consecuencia del primero, o respuesta colectiva a la exclusión ${ }^{4}$.

De ahí que las estrategias de exclusión sean el modo predominante de restricción en todo sistema estratificacional. En tanto en cuanto el grupo de los excluidos es capaz, a su vez, de cerrar el paso al resto de gratificaciones y oportunidades, multiplicándose así el número de subestratos, el modelo de estratificación social alcanza el máximo punto de contraste con el modelo marxista de la polarización entre las clases, a la vez que permite explicar sistemas concretos de desigualdad social en los que intervienen variables como las consideraciones étnico-raciales, más claramente.

De hecho, la idea de restricción se remite a la de poder, en el sentido weberiano del término. Las formas de restricción pueden considerarse como medios de movilizar el poder a fin de lidiar en la arena de la distribución. El poder puede concebirse como un atributo inherente a la restricción y, además, resulta consistente con el análisis de las relaciones de clase. De este modo, la distinción entre burguesía y proletariado, en su acepción clásica y revisada moderna, puede entenderse como la expresión del conflicto entre clases definido no específicamente en relación al lugar que ocupan en el proceso productivo, sino en relación a los modos de restricción prevalentes, a saber, la exclusión y la usurpación, respectivamente. Es también consistente con la tradición que sitúa el concepto de explotación en el centro del análisis de clase. Así, en la medida en que las formas excluyentes de restricción dan lugar al uso del poder hacia abajo, creando en consecuencia formaciones sociales subordinadas, pueden considerarse por definición como explotadoras. La explotación define el nexo entre clases u otras colectividades que se hallan en una relacción de dominación y subordinación, bajo cualquier base social. Parkin va más allá al asegurar que no hay razón para restringir el concepto de explotación al uso marxista convencional que lo refiere a la apropiación de plusvalía por parte del capital, puesto que éste no es más que un caso concreto del fenómeno más general de restricción excluyente.

De este modo, los esfuerzos colectivos para restringir el acceso a las recompensas y oportunidades por un grupo social en contra de otro,

4 F. PARKIN (1979), ibid., p. 48. Véase, también, F. PARKIN (1974), "Strategies of Social Closure in Class Formation", cn F. PARKIN (cd.), The Social Analysis of Class Structure, Tavistock Publications, Londres, pp. 1-18. 
inclusive un grupo de trabajadores frente a otro, puede contemplarse como inherentemente explotador incluso si la relación no es la de extraer plusvalía tal como deriva de la propiedad. Las relaciones de dominación y subordinación entre burguesía y proletariado, protestantes y católicos, blancos y negros, hombres y mujeres, etc., pueden considerarse como relaciones de explotación en el sentido neoweberiano. La objeción marxista a este uso amplio del término estriba en que viola el status científico del concepto 5 .

Sin embargo, aun en el caso argumentado por Parkin la propiedad sigue estando en el centro del análisis de la existencia de clases y de su división al determinar la explotación de un grupo social por otro, directamente a través de las instituciones legales de la propiedad, o bien indirectamente a través de las cualificaciones académicas y credenciales profesionales que controlan el acceso a los puestos clave en la escala jerárquica de la división del trabajo. Para Parkin, propiedad y conocimientos técnicos y profesionales (títulos académicos concretamente, según este autor) son los medios más extendidos de restricción explotadora y, por supuesto, excluyente en las sociedades avanzadas.

En realidad, la idea de explotación que deriva de la propiedad de los medios de producción, como central en el análisis de las clases, ha estado presente en la mayoría de las aportaciones de pensadores socialistas de éste y el pasado siglo. S. Ossowski arguye que sólo puede hablarse de sociedad sin clases si se elimina hipotéticamente la propiedad, de la cual depende la posesión de bienes y servicios y la distribución de poder y autoridad. Eliminar la dicotomía y desigualdad entre las clases sociales es posible hipotéticamente de acuerdo con una concepción saint-simoniana de producción y trabajo. Para este clásico, la clase de los productores incluye tanto a los industriales como a los asalariados sin propiedad. En esos términos podría hablarse de una sociedad sin clases compatible con la existencia de diferencias de poder y riqueza.

Empíricamente, no obstante, la representación de toda sociedad avanzada como un orden social vertical de divisiones o capas superpuestas lleva a Ossowski a intentar establecer los criterios y las modalidades de la gradación entre estas divisiones o capas. Así, en los "esquemas de gradación" que establece este autor pueden existir más de dos clases sociales antagónicas. Existen, de hecho, capas intermedias, las clases medias, que se reconocen por su ordenamiento y situación en la escala jerárquica del orden social vertical más que por sus relaciones de dependencia mutua. Es una concepción descriptiva más que explicativa del hecho de la desigualdad social y la división entre las clases.

Incluso en la concepción más simple de sociedad dividida en dos clases antagónicas pueden darse tres modalidades de dicotomía o antagonismo,

5 F. PARKIN (1979), op. cit., p. 50. 
fundamentadas en la distribución de privilegios en el reparto o distribución de beneficios. Las modalidades son tres. En primer lugar, existen los gobernantes y los gobernados: aquellos que mandan y aquellos que obedecen, divididos según los criterios de poder y autoridad, en términos de Dahrendorf. En segundo lugar, los ricos y los pobres: aquellos que poseen riqueza, en un sentido distinto al de propiedad, y aquellos que no la poseen. El criterio es aquí el de la diferenciación económica. En tercer lugar, aquellos para los que otras trabajan y aquellos que forman las clases trabajadoras. Esta separación pone de relieve la explotación de un grupo sobre otro, explotación que comporta la propiedad de los medios de producción ${ }^{6}$.

Estas tres modalidades representan la forma de división de clases dicotómica. No son mutuamente excluyentes, sino que pueden coincidir en el tiempo y en el espacio, aunque exista siempre una que sea dominante o predominante sobre las otras. Por otro lado, se pueden distinguir dos tipos de esquemas de gradación: el simple y el sintético. En el primero, la representación o imagen de la estructura de clases responde a un único criterio, la renta, por ejemplo. En el segundo se procede a un ordenamiento escalonado de clases según diversos criterios combinados, renta, status, privilegios, etc. En este caso, la concepción de clase es más laxa que la concepción clásica marxista. Existe una tercera forma de representación de las clases que Ossowski denomina "esquema funcional». Aquí el criterio principal de división de la sociedad en grupos o clases es la división del trabajo. Se supone que existe una pluralidad de clases, que no son antagónicas, sino que son interdependientes o cooperan entre sí. En esa tercera forma estarían representadas las clases medias en las sociedades avanzadas, tanto capitalistas occidentales como de socialismo $\mathrm{real}^{7}$.

Esta tercera forma da pie a argumentar sobre la sociedad sin clases si se hace hincapié en la dimensión psicológica del criterio de clase social, detectable a través de la opinión de la gente sobre el sistema de clases en el que viven y participan. El criterio de pertenencia a una u otra clase en combinación con el esquema funcional de gradación da como resultado la existencia de una amplia clase media en un país como los Estados Unidos. Esa constatación, corregible si se intruduce en las posibles respuestas la opción de "clase trabajadora", unida a la creencia generalizada de las oportunidades reales de movilidad ocupacional y social fruto del desarrollo industrial y económico, en general, da lugar a la imagen de la sociedad americana como "sociedad sin clases" o sociedad en la que existe "ausencia de clases".

Una matización importante al respecto es la que hace Lenski al con-

6 S. Ossowski (1963), Class Structure in the Social Consciousness, Routledge and Kegan Paul, Londres, p. 27.

7 S. OssowsKI (1963), ibid., p. 70. 
trastar los puntos de vista de distintos sociólogos norteamericanos al examinar el sistema de desigualdad social predominante en los USA. Según Lenski, por una parte, las clases sociales son consideradas como categorías estadísticas con una significación o importancia únicamente heurística; y, por otra parte, son contempladas como grupos sociales fundados en vínculos de tipo psicológico. La justificación de estos criterios estriba en afirmar que la escala de status social americana es un continuum asequible a todo individuo. El status económico y social de los individuos no está determinado por la herencia o el linaje y, a pesar de que todo el mundo no tenga el mismo punto de partida, la vía hacia posiciones más elevadas está abierta a todos. Además, la escala de status social no se rompe en tramos y barreras perceptibles que podrían transformar el continuum de posiciones de status en una gradación de estratos distintos. De acuerdo con ello, los privilegios que corresponden a cada eslabón de la escala no son definitivos, permanentes y perdurables, ni cerrados. En consecuencia, tampoco existe conflicto de intereses entre los niveles superiores e inferiores en términos de status social ${ }^{8}$.

\section{Distribución de autoridad $y$ de privilegios}

Lenski profundiza en la idea de privilegio como criterio central de la división en clases o grupos sociales de una sociedad. Su teoría de la estratificación social se fundamenta en el sistema de distribución de los bienes y servicios (excedente) que se producen en todas las sociedades. El análisis de la estratificación puede reducirse al examen del proceso distributivo en una sociedad según se reparta el control sobre alguna porción de ese excedente. A lo largo de la historia de la Humanidad, en toda sociedad, primitiva o moderna, se produce un excedente, que requiere, en última instancia, de un determinado nivel tecnológico. Así, en las sociedades primitivas, al ser bajo este nivel, la distribución del excedente de la actividad económica principal (la caza) se realizará según criterios de necesidades elementales, mientras que en las sociedades industriales modernas se habrá desarrollado un proceso productivo complejo (de acuerdo con el flujo de demandas crecientes y cualitativamente distintas) que hará preciso el posible control del proceso distributivo. El privilegio consiste en tener control sobre alguna porción del excedente de bienes y servicios creados en una sociedad. Depende fundamentalmente del poder o probabilidad de que una persona o grupo social pueda realizar su voluntad de ejercer ese control a pesar de o independientemente de que el resto de personas o del grupo se opongan a ello. El privilegio es, pues, función del poder ${ }^{9}$.

" G. LeNSKI (1952), "American Social Class: Statistical Strata or Social Group", American Joumal of Sociology, septiembre 1952, passim.

9 G. Lenski (1966), Power and Privilege, McGraw Hill, Inc., Nueva York y Londres, Pp. $16-21$ 
El concepto de privilegio es un instrumento heurístico útil para analizar la realidad de otro bloque de sociedades avanzadas: las de socialismo real. También en ese caso se ha argumentado sobre la desaparición de la división de clases sociales, aunque persisten diferencias importantes en la participación en la renta nacional. Cabría hablar aquí igualmente de un credo comunista paralelo al credo americano sobre la igualdad de oportunidades, fundado en la creencia de "a cada cual según sus necesidades". El status de un individuo estaría condicionado por sus propias capacidades de alcanzar posiciones superiores en la escala ocupacional y social o en los propios méritos condicionados por el tipo de trabajo o actividad asignados. En un sociedad como la Unión Soviética existen privilegios económicos y discriminaciones sociales, incluso diferencias de ingresos que permiten apuntar a la aparición de una nueva clase intermedia: la clase social privilegiada, la intelligentsia o la meritocracia. Con todo, la desaparición del antagonismo entre las clases (por la desaparición de la propiedad privada de los medios de producción) y la aparición de una escala de status social, permite constatar la existencia de grupos sociales privilegiados y no privilegiados, de acuerdo con una concepción de clase social más próxima a la de Adam Smith que a la de Karl Marx ${ }^{10}$.

Desde una concepción próxima a la marxiana, Wesolowski muestra cómo la relación con los medios de producción determina, a través de un conjunto complejo de interrelaciones, los atributos de la posición de clase como son la renta, el trabajo, el prestigio, las oportunidades de vida. En las sociedades socialistas se tiende a uniformar la relación de la posición de clase con los medios de producción de modo que éstos pierden sus propiedades determinantes. Pero los atributos de la posición social continúan diferenciando a los grupos sociales que adquieren cierta autonomía bajo control, en el socialismo, por la desvinculación del status en relación a la propiedad y al poder ${ }^{11}$.

De ahí que el conflicto en las sociedades socialistas tenga su origen en la distribución de bienes y servicios de distinta naturaleza y sea susceptible, por tanto, de control de acuerdo con las políticas sociales de gobierno. Ello es debido al principio regidor de "a cada cual según su trabajo", principio que asegura tanto el consensus societal y los medios apropiados para la transición al estadio en el que el principio de "a cada cual según sus necesidades" se convierte en realidad. La diferencia socioocupacional más que la propiedad es el origen de la contradicción estructural de intereses en la sociedad socialista, fuente de tensión y conflicto, que, lejos de anular las realizaciones de unos u otros grupos, moviliza los recursos humanos y materiales de la sociedad. En este sentido, Wesolowski considera que las clases pueden haber desaparecido pero persiste la estratificación social en las sociedades socialistas.

10 S. OssowsKI (1963), op. cit., p. 184.

11 W. WesolowsKi (1979), Classes, Strata and Power, Routledge and Kegan Paul, Londres, pp. XVII-XX. 
Influido, tal vez, por su propia crítica a la teoría funcionalista de la estratificación social ${ }^{12}$, Wesolowski define la estratificación como el sistema de posiciones sociales que tienen una distribución desigual de un valor fundamental: la autoridad. En las sociedades industriales que difieren en su organización social (las capitalistas y las socialistas) pude ocurrir que su estructura interna de valores difiera. La diferencia consiste no sólo en el diferente "peso" dado a los distintos valores como un sistema total, sino tal vez en el "carácter" diferente de cada valor en particular. Uno de esos valores es que en las sociedades avanzadas las posiciones de autoridad proporcionan a los individuos una gran oportunidad de manifestar su propia personalidad, su talento, sus ideas, sus habilidades, aparte de la posibilidad de satisfacer su afán de poder y su deseo de mandar o dirigir a otros individuos en posiciones subordinadas.

En este sentido, en las sociedades avanzadas, las motivaciones de las personas para cubrir posiciones socialmente importantes, actividades adscritas a esas posiciones que requieren un nivel de especialización elevado y un grado de educación apropiado no son necesariamente las recompensas materiales y el prestigio (como dicen los sociólogos funcionalistas). La diferenciación de ventajas materiales puede no ser una «necesidad funcional». Tanto las posiciones que requieren educación y especialización como las posiciones de autoridad deben ser cubiertas no porque of rezcan ventajas materiales, sino porque sus atributos principales: habilidad, conocimientos y poder, resultan ser suficientemente atractivos. Si se acepta que el deseo de tener formación o educación (conocimientos, habilidades) y el deseo de poseer autoridad actúan como motivaciones conducentes a la ocupación de posiciones sociales importantes que, a su vez, requieren de la especialización de las personas que desempeñan las actividades adscritas a tales ocupaciones, el prestigio derivará de las posiciones que conlleven mayor o menor nivel de educación y autoridad. Las diferencias en educación y en autoridad, que no desaparecen totalmente en ninguna sociedad, originan estimaciones distintas por lo que se refiere al prestigio de las posiciones que tienen elementos variantes de habilidad y poder en el desempeño de sus funciones. En conclusión, para Wesolowski, la autoridad, más que las recompensas materiales o el prestigio, es el elemento de estratificación más determinante, a diferencia de la argumentación funcionalista.

12 En 1962, Wesolowski publicó una excelente crítica a las posiciones de Davis y Moore. Véase "Some notes on the functional Theory of Stratification", The Polish Sociological Butlletin, núms. 3-4 (5-6); reproducido en C. S. Heller (ed.), Structured Social Inequality, MacMillan, Londres y Nueva York, 1969. 
Esquemas relacionales o gradacionales

El énfasis puesto por Wesolowski en la educación, conocimientos y habilidades, le lleva a prestar atención al desarrollo de un grupo social, la intelligentsia, de auge importante en las sociedades socialistas. Este grupo social es un estrato y no una clase. No se distingue ni define por su relación antagónica con otro grupo social sino que históricamente ha estado vinculada a las clases dominantes, no sólo bajo el capitalismo, sino también, en cierta medida, en formaciones económico-sociales precedentes. Como grupo social, se puede generalmente identificar a la intelligentsia por una serie de atributos como son: el tipo de trabajo no-manual, los niveles de renta y prestigio, una cierta forma de posición política "indefinida" por lo que se refiere a los conflictos de clase. Entre los atributos descriptivos, y hasta cierto punto, definitorios, de intelligentsia no se incluye el criterio fundamental de la definición clásica de clase social, a saber, las relaciones de un grupo social con los medios de producción. Si así se hiciera, podría considerarse a ese grupo social en relación antagónica con otro grupo o clase social y podría identificarse como clase explotadora, dominante, o bien, clase explotada o subordinada. En realidad, los atributos que identifican a la intelligentsia manifiestan la posición social del grupo social más que sus relaciones con los medios de producción ${ }^{13}$.

Entre esos atributos, el más significativo es el de la naturaleza nomanual del trabajo que realizan los miembros de la intelligentsia. En la sociedad capitalista, la intelligentsia subsume tanto a los empleados como a profesionales, trabajadores autónomos y profesionales empleados en instituciones públicas. Bajo el socialismo, profesionales y trabajadores son todos ellos empleados. Debe reconocerse, asimismo, la tendencia a la concentración y centralización de los medios de producción en muchas sociedades capitalistas contemporáneas, que lleva a gran parte de los profesionales liberales a ocuparse en las grandes organizaciones o instituciones, así como la tendencia bajo el socialismo a subsumir las áreas de la producción y de los servicios en un sector socializado.

Bajo el capitalismo, la clase trabajadora y la intelligentsia se definen de acuerdo con dos marcos de referencia distintos: el sistema económico de explotación y la ubicación en la división socioocupacional del trabajo, respectivamente. En el socialismo, las respectivas ubicaciones se pueden analizar según el mismo marco de referencia, en especial la división socioocupacional del trabajo. Ya no es la relación con los medios de producción, en el caso de los trabajadores, o la naturaleza del trabajo, en el caso de la intelligentsia, lo que les ubica en una u otra posición de la división del trabajo, sino el propio tipo de atributos, que pueden ser contradictorios y gradacionales. Trabajadores e intelligentsia tienen, bajo el socialismo, una carac-

13 W. WesolowsKi (1979), op. cit., p. 108. 
terística común: ambos grupos son empleados, ocupados, en el sector socializado de la economía. Difieren únicamente por el carácter o naturaleza del trabajo: manual o no-manual, realizado; los niveles de renta, educación y prestigio.

Este es un paso importante en el esfuerzo por hacer las posiciones sociales más homogéneas. Una disminución en las diferencias de renta, educación y prestigio y las proporciones diferenciales de trabajo manual y no-manual en la actividad adscrita a una ocupación (principalmente a través del incremento en el elemento de la actividad mental en la realización de un trabajo por parte de los trabajadores manuales) tiende hacia la dirección de disminuir las diferencias de naturaleza de clase en contraposición a estrato, entre estos grupos sociales. En el curso de su desarrollo, los atributos identificadores de clase o estrato se transforman en gradacionales, perdiendo su carácter de contradictorios. Las nuevas tecnologías y nuevas formas de gestión eliminan, en muchos casos, las distinciones entre actividad manual y no-manual, manager y managed en el proceso de trabajo ${ }^{14}$.

Ahora bien, aunque la intelligentsia no constituye una clase social de acuerdo con los criterios de propiedad o herencia de riqueza, ni constituye una élite en el sentido de grupo cerrado al que no existe acceso alguno por movilidad ascendente, es un grupo social privilegiado, con un consagrado interés en maximizar su poder redistributivo como administrador del excedente social. Por encima de las divisiones y jerarquías existentes en su seno, los miembros de la intelligentsia comparten un compromiso común con el Estado socialista y un sentido de su propia interdependencia en alcanzar objetivos societales que refuercen su cohesión y poder. A pesar de la abolición de la institución de la propiedad privada de los medios de producción, los trabajadores son una clase subprivilegiada desprovista de autodeterminación o control sobre el excedente. Los salarios diferenciales, la explotación económica y la alienación por el trabajo, constituyen problemas estructurales acuciantes en las sociedades socialistas, al igual que en las capitalistas ${ }^{15}$.

Persisten las situaciones reales y concretas de relaciones de dominación-subordinación entre los grupos sociales. Tres tipos de teoría han intentado explicarlas en relación a la división social de poder. En primer lugar, deben tenerse en cuenta las diversas versiones de la teoría marxiana de las clases dominantes y subordinadas; en segundo lugar, las diferentes teorías de las élites de poder y las masas; y en tercer lugar, las diversas versiones de la teoría de los grupos-de-intereses. Desde perspectivas distintas, cada una de esas teorías trata de describir y explicar la división de poder en una sociedad estableciendo los puntos de contraposición, compe-

14 W. WESOLOWSKI (1979), op. cit., pP. 110-111.

15 G. KonRAD e I. SZÉLÈNY (1980), The Intellectuals in the Road to Class Power, Haverster, Brighton, p. 78. 
tencia e, incluso, contradicción entre los grupos sociales. Pero, desde la perspectiva de Wesolowski, se trata de mostrar que estos tres tipos de teoría ponen el énfasis sobre diferentes fenómenos o sobre distintos aspectos del mismo fenómeno y, en consecuencia, pueden contemplarse, hasta cierto punto, como complementarios.

Wesolowski muestra la complementariedad de dos posiciones teóricas distintas. La primera, ganado predicamento entre la sociología occidental contemporánea, examina y analiza la élite de poder aisladamente de la estructura de clases en el sentido marxista del término. La élite de poder, según esta visión, consiste en las personas que toman decisiones políticas, en la conexión entre ellas y sus decisiones. La estructura de clases es relegada a una posición secundaria en ese planteamiento. La segunda posición ha predominado hasta muy recientemente en la sociología marxista. En el marco de la estructura de clases la élite se engloba e, incluso, se identifica como sinónimo de "clase dominante". Aun cuando pueda considerarse a la élite como una colectividad separada, su único rol sería el de transmitir la voluntad de clase y servir como representante de sus intereses.

En realidad, ambas posiciones pretenden ignorar la aportación de Marx. De acuerdo con la teoría marxiana, la clase dominante es producto del sistema económico. Se compone de aquellos individuos que, por su relación con los medios de producción, ocupan una posición privilegiada en el proceso de distribución del producto social. En un sistema capitalista, la clase dominante sería el grupo de los capitalistas. La dominación social de éstos consiste en su control sobre los medios de producción, el proceso productivo y el producto del trabajo, por una parte; $y$, por otra, el hecho de que las normas legales fundamentales del Estado (la Constitución y otras leyes) garantizan ese control, justificado y legitimado por la ideología dominante. De ahí que, en términos marxianos, cabe hablar de dominación económica, dominación política y dominación ideológica.

La élite de poder, en cambio, es el producto de la estructura política de la sociedad. Engloba a aquellos individuos que participan en el proceso de toma de decisiones por el Estado. Su poder depende de su habilidad para tener influencia directa sobre quienes toman decisiones, o bien sobre la real participación en el propio proceso de toma de decisiones. La distinción entre la influencia sobre el proceso de toma de decisiones y la participación efectiva en el proceso se une a la existencia de estructuras políticas y personas distintas especializadas en cada una de estas dos funciones en los Estados contemporáneos.

Las decisiones se toman en las tres ramas de poder: legislativo, ejecutivo y judicial. Estructuras fomales, como los partidos políticos y los grupos de intereses, ejercen influencia externa sobre estas decisiones; juntamente con numerosas estructuras informales como las asociaciones de diversa indole. Por esta razón puede afirmarse que la élite de poder es el producto 
tanto de estructuras políticas formales como informales de la sociedad. Según el contenido y la forma de actividad, las élites serán de decisión o de influencia ${ }^{16}$.

La distinción entre clase y élite es útil a efectos analíticos a la hora de definir con precisión los conceptos como instrumentos heurísticos y las relaciones entre las realidades concretas a las que se refieren o contraponen (grupos sociales, masas). Por otro lado, la distinción entre clase social y estrato se halla en la base de muchos planteamientos teóricos sobre la estructura social y la estraficación (esquemas de gradación para Ossowski, restricción excluyente para Parkin, etc.). En tercer lugar, cabe la distinción entre evaluación de la división y diferenciación entre clases sociales según criterios objetivables (el de renta o ingresos es el más evidente) o no, al precisar los límites de cada grupo social. Así, difícilmente pueden establecerse las fronteras entre distintas clases sociales, altas, medias o bajas; burguesía o proletariado, capitalistas o trabajadores, siguiendo únicamente criterios objetivables y, en última instancia, mensurables.

Ello pone sobre el tapete la importancia de las relaciones entre las clases más que la naturaleza de las mismas o la aplicación de ese concepto. Esa es la tarea de Giddens al poner el énfasis en la necesidad de conceptualizar la estructuración de las relaciones de clase, es decir, los procesos a través de los cuales las "clases económicas" se convierten en "clases sociales" y éstas, a su vez, se relacionan con otras formas sociales. La estructuración de las relaciones de clase implica poner el acento en los modos en que las relaciones económicas se traducen en estructuras sociales no-económicas $^{17}$.

Partiendo de la distinción entre estructuración mediata (mediate) e inmediata (proximate), a saber, los factores que intervienen en la existencia de determinadas capacidades de mercado en conexión con la formación de las clases como grupos sociales identificables (o sea, las conexiones entre el mercado y el sistema estructurado de relaciones de clase), por un lado, y, por otro, los factores "localizados" que condicionan o dan lugar a la formación de las clases (a saber, la división del trabajo en el seno de una empresa, las relaciones de autoridad en ella, la influencia de los ugrupos distributivos"), puede afirmarse que la estructuración de las clases se produce en la medida en que existe la restricción a la movilidad en relación a toda forma específica de capacidad de mercado.

Hay tres formas de capacidad de mercado: la propiedad de los medios de producción, la posesión de cualificaciones técnicas y educación, la posesión de fuerza de trabajo manual. En tanto en cuanto estas formas se vinculen a formas cerradas de movilidad inter e intrageneracional, puede hablarse de la fundación de un sistema básico de tres clases sociales en la

16 W. WESOLOWSKI (1979), op. cit., pp. 49-52.

17 A. Giddens (1973), The Class Structure of the Advanced Societies, Hutchinson University Library, Londres, p. 97. 
sociedad capitalista: la clase alta, la media y la baja o trabajadora. Ahora bien, al ser inherente al capitalismo que no existan barreras legalmente establecidas o limitaciones formalmente prescritas a la movilidad, no puede afirmarse que se produzcan restricciones completas o totales a las oportunidades de movilidad, cuya distribución está en la base de la estructuración mediata de las relaciones de clase. Es, por lo tanto, obligado volver los ojos hacia las fuentes de la estructuración inmediata.

La división del trabajo es un elemento clave en la fragmentación y, a la vez, consolidación de las relaciones de clase. Promueve la formación de clases en la medida en que crea grupos homogéneos que se aúnan en las sociedades industriales avanzadas según la disponibilidad de tecnología. La utilización de la técnica, en un sentido amplio y actualmente representada por los sistemas cibernéticos de control, crea una separación decisiva entre las condiciones laborales y productivas de los trabajadores manuales y no manuales. Este efecto se combina con el de la distribución de autoridad en el lugar de trabajo, que refuerza la separación de los trabajadores que participan en las tareas de control (los administrativos), separándolos de los trabajadores manuales. Refuerza, asimismo, la separación de ese grupo de quienes se sitúan en la cúpula del sistema de autoridad en la empresa o lugar de trabajo, en razón de poseer los medios de producción. Así, puede distinguirse la clase alta de la clase media.

La tercera fuente de estructuración inmediata de clases se encuentra en la esfera del consumo más que de la producción. Las pautas de consumo influyen en la estructuración de las clases, de modo que existen "grupos distributivos" cuyas relaciones implican pautas comunes de consumo de bienes económicos, independientemente de la evaluación por criterios de honor o prestigio de los individuos que componen el grupo, en términos weberianos; y grupos de status que toman como punto de referencia tales evaluaciones. Los "grupos distributivos" son importantes en la estructuración de las relaciones de clase por cuanto se interrelacionan con los demás factores de modo que refuerzan las separaciones entre formas de capacidad de mercado. Un ejemplo de ello es la tendencia a la segregación por comunidades o barrios según criterios como el nivel de ingresos o la posibilidad de obtener créditos para la vivienda (barrios obreros, de clase media, etcétera $^{18}$.

En resumen, en la medida en que coincidan las bases sobre las que se fundamentan la estructuración de clases mediata e inmediata, existirán clases sociales como formaciones claramente distinguibles. La combinación de las fuentes de la estructuración mediata e inmediata da lugar a una estructura de clases tripartita genérica a la sociedad capitalista. Ahora bien, el modo en que estos elementos se combinan para formar un sistema de clases específico, en cualquier sociedad dada, difiere significativamente según

18 A. GIDDENS (1973), ibid., pp. 107-111. 
las variaciones en su desarrollo económico y político. Por otra parte, sólo empíricamente pueden establecerse los límites entre las distintas clases sociales una vez establecido el modo de estructuración que las enmarca.

\section{Grupos sociales en los intersticios de las clases}

La cuestión de los límites o fronteras entre las clases sociales es analizada por Wright. A fin de obviar las ambigüedades que puedan presentarse en la definición de los grupos sociales es preciso contemplar algunas situaciones ocupando posiciones (locations) objetivamente contradictorias dentro de las relaciones de clase. Lejos de erradicar esa contradicción clasificando artificialmente cada posición en la división social del trabajo como una u otra clase, sin ambigüedades, deben analizarse las posiciones contradictorias. Ciertamente, toda posición de clase es una posición contradictoria, puesto que las relaciones entre las clases son intrínsecamente antagónicas, son relaciones sociales contradictorias. Pero determinadas posiciones en la estructura social constituyen contradictory locations dobles al ser posiciones contradictorias en el seno de las relaciones de clase antagónicas básicas.

A fin de poder explicar en su globalidad la estructura de clases de las sociedades capitalistas dando cuenta de las posiciones contradictorias que se generan en su seno, deben comprenderse los diversos procesos que constituyen las relaciones de clase, analizar su transformación histórica en el curso del desarrollo capitalista y concluir de todo ello cómo derivan las posiciones contradictorias de la diferenciación de estos procesos. A partir de ahí, Wright concluye que existen tres grupos de posiciones dentro de la división social del trabajo que pueden caracterizarse como posiciones contradictorias en el seno de su relaciones de clase. Esos grupos son: los managers y supervisores que ocupan una posición contradictoria entre la burguesía y el proletariado; determinadas categorías de empleados semiautónomos que mantienen niveles de control relativamente elevados sobre el proceso de trabajo que les resulta más inmediato y ocupan una posición contradictoria entre la clase obrera y la pequeña burguesía; los pequeños empleadores que ocupan una posición contradictoria entre la burguesía y. la pequeña burguesía. Estas posiciones contradictorias surgen de la dinámica de las relaciones de clase en las sociedades avanzadas. Así, en el caso de las clases medias podría derivar su posición contradictoria entre las clases altas y bajas (burguesía y pequeña burguesía) de su doble función y situación respecto al capital y al trabajo colectivamente organizado ${ }^{19}$.

Los cambios estructurales que, de forma interconectada, se han produ70.

19 E. O. WRight (1978), Class, Crisis and the State, New Left Books, Londres, pp. 61- 
cido a lo largo del desarrollo del capitalismo son fundamentalmente los siguientes. En primer lugar, la progresiva pérdida de control sobre el proceso productivo por parte de los productores que intervienen en él directamente. En segundo lugar, la formación de complejas jerarquías de autoridad y división de funciones originalmente atribuidas al capitalista empresario. En tercer lugar, la diferenciación de estas funciones en conexión con la estructura jerárquica de autoridad en las empresas capitalistas y organizaciones burocráticas ${ }^{20}$. Estos cambios condicionan los procesos sociales subyacentes a las relaciones de clases en las sociedades capitalistas avanzadas, pero no coinciden siempre y de la misma manera. Esta no coincidencia es lo que determina las posiciones contradictorias en el seno de las relaciones de clase.

Por lo que respecta a las posiciones contradictorias entre la pequeña burguesía y las demás clases sociales, el proletariado y la burguesía, deben considerarse las posiciones respectivas entre el modo de producción capitalista y la producción de mercaderías simple, esto es, entre dos modos de producción distintos. El rasgo característico de la producción capitalista es la apropiación de plusvalía a través de la explotación de los trabajadores en el proceso productivo. En la producción simple de mercaderías no existe explotación, toda plusvalía es generada por el productor pequeño burgués y por su familia. A pesar de que éste puede emplear a algún o algunos trabajadores, la porción de plusvalía generada por éste o éstos será progresivamente menor que la generada por la familia pequeño burguesa hasta que el número de empleados sea relativamente elevado. Aunque no pueda determinarse el número de empleados necesario para producir esa inflexión, el pequeño burgués se convierte, llegado este punto, en pequeño capitalista. Ahí, la posición contradictoria entre la clase capitalista burguesa y la pequeña burguesía.

En cuanto a la posición contradictoria entre la pequeña burguesía y el proletariado, el control sobre el proceso productivo es la pieza clave para entender la transformación de la pequeña burguesía en clase trabajadora en virtud de la penetración del dominio capitalista en la organización del proceso de producción que lleva a perder todo control por parte del productor directo. Hay, no obstante, categorías de empleados que mantienen cierto control, a veces elevado, sobre sus condiciones de trabajo y el proceso productivo; controlan cómo hacen su trabajo y qué bien o servicio producen (por ejemplo, un profesor universitario, funcionarios de corporaciones). El criterio de semi-autonomía en el desempeño de la actividad productiva individual, aun participando en una organización colectiva del trabajo, implica un mínimo control sobre lo que se produce (propiedad económica mínima), así como sobre cómo se produce (posesión mínima) ${ }^{21}$.

20 E. O. WRIGHT (1978), ibid, cap. 2.

21 E. O. WRIGHT (1978), ibid, pp. 78-83. 
Esa diferenciación, paralela a la distinción entre propiedad y control de los medios de producción, avala el criterio de semiautonomía como útil para analizar y, si cabe, definir las posiciones sociales correspondientes a actividades derivadas del desarrollo del capitalismo avanzado, actividades que se adscriben a ocupaciones nuevas desempeñadas por personas que se autodenominan pertenecientes a las clases medias en las sociedades avanzadas.

Al intentar definir las clases medias, algunos autores recurren a criterios complementarios al elemento clásico de la propiedad, como son los conceptos de autoridad (Dahrendorf), conocimientos técnicos y científicos (Parkin), privilegio (Lenski), prestigio y autoridad que conlleva un determinado grado de educación (Wesolowski). Paralelamente, se establecen esquemas gradacionales (Ossowski, Wesolowski), de restricción excluyente (Parkin) y otros, para dar cabida a las clases medias entre las clases sociales dicotómicas. Finalmente, se tienen en cuenta las relaciones entre las clases y su estructuración (Giddens), además de considerar como centrales las posiciones contradictorias en que se encuentran diversos agrupamientos humanos en las sociedades avanzadas (Wright).

Todos estos elementos de análisis aumentan considerablemente la posibilidad de comprender la compleja estructura social de las sociedades avanzadas reforzando la viabilidad del concepto de clases medias para examinar empíricamente la realidad de esas sociedades. Desde el marco general de las teorías de la convergencia de las sociedades industriales (Kerr, Dunlop) o la posibilidad de la abolición de las clases (Marx) o la consecución de una sociedad más igualitaria (Tocqueville) o con diferencias y desigualdades menos abruptas (Wesolowski), pueden analizarse las matizaciones y perfiles que toman los grupos sociales en las sociedades en las que el conocimiento científico y técnico se ha convertido en un elemento central de su desarrollo económico y social. Sin profundizar en las consecuencias políticas de tales transformaciones estructurales en el proceso y forma de producir bienes y servicios, en la organización de ese proceso y en el peso que progresivamente adquiere el sector servicios (en un sentido amplio) en las sociedades avanzadas, es importante señalar la imbricación entre grupos sociales, en teoría y en principio, distinguibles en esas sociedades. La estructura social de muchas sociedades contemporáneas, incluida la nuestra, presenta como una de las características más relevantes y notorias el crecimiento de las llamadas clases medias. La profusión de literatura sociológica sobre ese tema recientemente en España da cuenta del interés que suscita entre los estudiosos 22 la realidad de una estructura social que

22 Sirvan de ejemplo, sin ánimo de pretender ser exhaustivos, los siguientes trabajos: J. F. Tezanos (1973), Las nuevas clases medias, Cuadernos para el Diálogo, Madrid; D. LACAlle (1982), Los trabajadores intelectuales y la estructura de clases, CIS, Madrid; T. MOLTÓ (1979), "Els treballadors intel.lectuals en la producció capitalista", Nous Horitzons, núm. 58, noviembre 1979; J. Crespán (1979), en J. F. Marsal y B. Oltra (cds.), Nuestra Sociedad, Ed. Vicens Vives, Barcelona, cap. "Las clases medias"; L. CARreño, 
ha evolucionado profunda y rápidamente en los últimos cincuenta años. Queda, sin embargo, insatisfacción a la hora de decidir utilizar uno u otro/os criterios de definición para establecer los límites de las clases respecto del resto de clases sociales.

La dificultad está, tal vez, en tener que abandonar los planteamientos de "suma cero" al abordar la realidad de la estructura social de las sociedades contemporáneas. Ello obliga a matizar, o incluso complementar, un esquema de clases dicotómico con un continuum estratificacional o gradacional más que relacional. Así, no puede hablarse en términos excluyentes de clases sociales antagónicas, sino de estratos en una estructura ocupacional, de poder y de autoridad.

Eso nos lleva a considerar la multiplicidad de criterios para analizar la estructura social de nuestras sociedades. Si en el fondo del análisis de Marx y de pensadores marxistas la idea de explotación es básica en conexión con la posibilidad de apropiarse de los medios de producción por parte de un grupo en detrimento de otro (en un esquema de "suma cero") y con la existencia de clases antagónicas, algunos autores contemporáneos dan prioridad al concepto de dominación, en el sentido de expansión de la acción social, sobre el de explotación (Wrigth), como determinante de las diferencias entre grupos sociales. Otros autores (Parkin, por ejemplo) amplían el concepto de explotación a toda relación desigual de poder. Al no poder restringirlo a la propiedad de las fuerzas productivas o medios de producción, como se deriva de un análisis marxista de la realidad, el concepto de explotación queda desdibujado en sus contornos.

Si, además, se contemplan criterios como el de privilegio o prestigio, más que el de la propiedad de los medios de producción, para entender las diferentes posiciones sociales de los grupos o clases en una escala o continuum gradacional, la estructura social no se plantea ya en términos autoexcluyentes, sino que los grupos sociales se hallan ubicados en dos o más posiciones sociales de acuerdo con los dos o más criterios que definen su

O. Homs y M. Subirats, "Per una tipologia de les capes mitjanes", en S. AGuilar y otros, Visió de Catalunya, Diputació de Barcelona, Barcelona, 1987; F. MiguÉlez y C. SOlÉ (1987), Classes socials i poder politic a Catalunya, PPU, Barcelona, y S. DEL CAMPO (1989), La sociedad de clases medias, Espasa-Calpe, Madrid. Influencia decisiva han tenido obras publicadas recientemente en España que sitúan la necesidad de conceptualizar las llamadas clases medias en el marco del debate sobre el impacto de las nuevas tecnologías en el proceso productivo y nueva estructura ocupacional, así como el debate sobre el desarrollo de la socialdemocracia en el conjunto europeo, sin distinción de regímenes políticos. Sirvan de ejemplo las siguientes aportaciones: J. F. TEZANOS, R. CÓTARElo y A. DE Blas (1989), La transición democrática española, Ed. Sistema, Madrid; M. CASTELls et al. (1986), Nuevas tecnologias, economia y sociedad en España, Alianza Ed., Madrid; J. I. GershUNDY e I. D. MILES (1988), La nueva economia de servicios, Ministerio de Trabajo y Seguridad Social, Madrid; J. F. Tezanos (1987) (ed.), La democratización del trabajo, Ed. Sistema, Madrid; J. E. RoDríGUEZ IBÁÑEZ, "De la sociedad del trabajo a la sociedad tecnológica", REVISTA ESPAÑOLA DE

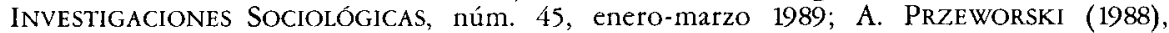
Capitalismo y socialdemocracia, Alianza Ed., Madrid, y O. LAFONTAINE (1989), La sociedad del futuro, Ed. Sistema, Madrid. 
situación real en las esferas de la producción y distribución de bienes y servicios. Existen, pues, múltiples situaciones intermedias en las que coinciden grados diversos de control sobre los medios materiales de producción y sobre la asignación de recursos (relaciones de apropiación, propiedad) y de control sobre la fuerza de trabajo (relaciones de dominación, autoridad).

Por otra parte, siendo el problema de la desigualdad social central en el análisis sociológico de la realidad, es relevante que en el examen de su plasmación en la estructura social el tema de la expansión de las clases medias en las sociedades capitalistas y las capas privilegiadas en las sociedades socialistas se plantee en el marco ideológico de la superación o abolición del sistema de desigualdad social correspondiente. La correlación entre la constatación empírica de la expansión de las clases o capas intermedias y la premisa de una sociedad sin clases está implícita en muchos análisis sobre la estructura social de las sociedades avanzadas.

En todo caso, es importante dar contenido a un concepto en proceso de revisión teórica a partir del creciente número de estudios empíricos sobre la compleja estructura social de las sociedades avanzadas, el análisis comparativo de los datos que se obtienen de su descripción y la reflexión a través de los autores, clásicos y contemporáneos, que han contribuido al conocimiento de la desigualdad social y su plasmación en la división, salvable o inevitable, entre los agrupamientos humanos en general y los grupos sociales en concreto. 